an abrupt increase in amplitude as the diameter decreases below $200 \mathrm{~km}$ (to about $0.21 \pm 0.01$ ). Even so, the shapes of smaller asteroids do not become significantly more elongated with decreasing size.

A search for possible collision-induced effects revealed that asteroids found in the zones of the asteroid belt which have low number densities do seem to have a higher value for the amplitude variation. Unfortunately the relative numbers of the specific asteroid types vary as one moves from zone to zone and this further complicates the picture.

When it comes to asteroid families it seems that the smaller members of the families have higher spin rates than $<175 \mathrm{~km}$ ) are virtually indistinguishable from non-family asteroids.

The significant conclusion of this investigation is that there is a pronounced normal. Larger family members $(75<D$

change in asteroid rotational properties at about $200 \mathrm{~km}$. Asteroids larger than this seem to be primordial (at least in the sense that they are not remnants of a larger disrupted parent body), nearly spherical and probably have prograde rotation. Smaller asteroids have random spin axis orientations and the population contains a higher proportion of slowly rotating objects. It is interesting to note that the dividing diameter of $200 \mathrm{~km}$ is strongly evident when the frequency-size relationship of asteroids is investigated. The mass distribution index of asteroids changes at this diameter.

Future progress in the study of asteroid spin statistics and the effect of collisional evolution depends on the acquisition of more data. The picture will be a lot clearer if the number of asteroids with accurately measured spin periods could be increased by a factor of two or three.

\section{Production and processing of prolactin}

\author{
from Alan D.B. Malcolm
}

THE pituitary hormone prolactin (PRL) was for many years something of an enigma. Its similarity to growth hormone both chemically (human PRL and human $\mathrm{GH}$ have 40 per cent of their amino acids in common) and biologically (in lower mammals PRL acts as a growth hormone) meant that its very existence in humans was disputed. Advances in protein purification and radioimmunoassay have now resolved this question and we are beginning to understand something about the factors controlling its synthesis and breakdown. Controls seem to exist at many stages including transcription, RNA processing, translation, preprolactin processing and secretion together with nuclease degradation of RNA and proteolytic attack on the various polypeptides.

The mRNA for rat PRL was obtained by extracting the poly(A)-containing RNA from the pituitary after stimulation of the rat with diethylstilboestrol (Gubbins et al. Nucleic Acids Res. 6, 915; 1979). This RNA was used to produce cloned copy DNA, which has since been used to identify genomic PRL sequences in a rat genome 'library'. The PRL gene contains four intervening sequences (Chien and Thompson Proc. natn. Acad. Sci. U.S.A. 77,$4583 ; 1980$ ), one of which occurs in the 5 -untranslated region. The sequence of one of the genomic clones (Gubbins et al. J. biol. Chem. 255, 8655; 1980) shows that some of the intron sequences are repeated elsewhere in the rat genome. It is interesting that although the coding lengths of PRL and $\mathrm{GH}$ are similar and they both have four introns, the lengths of the introns are much greater in PRL than in GH. The total length of the PRL 'gene' is therefore 10 kilobases compared with only 2.1 for GH.

Maurer and his colleagues $(J$. biol. Chem. 255, 2243; 1980) have approached the problem of transcription and processing of PRL RNA by isolating RNA from the nucleus and cytoplasm of pituitary cells. The RNA was then electrophoresed through an agarose gel and transferred to diazobenzyloxymethyl paper. Hybridization of the now immobilized RNA to ${ }^{32} \mathrm{P}$-labelled cDNA revealed the presence of five PRL bands $(7.0,6.4,3.8,1.7$ and 1.0 kilobases) in nuclear RNA but only one (1.0 kilobase) in cytoplasmic RNA. Presumably the different nuclear RNA species arise from ordered removal of the introns.

The secretion of all the pituitary hormones is controlled by the hypothalamus. In the case of PRL, dopamine is probably an important inhibitory factor and many dopamine agonists, such as the ergot alkaloids ergocryptine (EC) and its synthetic congener bromoergocryptine (BEC), will reduce overall PRL production. EC is used clinically to reduce PRL levels in spite of slightly unpredictable side effects (New Engl. J. Med. 302, 749; 1980).

To investigate the stage at which $\mathrm{EC}$ regulates PRL production, Maurer ( $J$. biol. Chem. 255, 8092; 1980) has studied the decrease in both de novo PRL synthesis

Alan D.B. Malcolm is in the Biochemistry Department at St Mary's Hospital Medical School, London. and PRL mRNA in cultured pituitary cells after treatment with EC. The very good correlation between the two suggests that EC acts either at transcription, RNA processing or RNA breakdown. It is slightly odd that the decreases occur over 20-100 hours which suggests that the mRNA may have a fairly long half life. Interestingly though, EC affects PRL specifically (no other pituitary hormones are affected) and is 100 times more effective than is dopamine.

This picture is, however, somewhat confused by the finding that $\mathrm{BEC}$ increases the rate of breakdown of the hormone itself (Dannies and Rudnick J. biol. Chem. 255, 2776; 1980). Maurer (Biochemistry 19, $3573 ; 1980$ ) suggests that BEC blocks PRL secretion by the cells and the subsequent accumulation inside the cells results in increased proteolysis by lysosomal enzymes. The fact that chloroquine (which reduces the rate of appearance of lysosomal enzymes in the cytoplasm) partially reverses this effect of BEC favours such an interpretation.

Hormone production can also be prevented by incubating the pituitary cells with hydroxynorvaline - an analogue of threonine which can be incorporated into protein. Normal processing of preprolactin to PRL involves cleavage at the bond between residues 30 (normally threonine) and 31. Apparently the processing enzyme cannot hydrolyse this bond when threonine is substituted by hydroxynorvaline because the prehormone accumulates in the analogue-treated cells (Hortin and Boime J. biol. Chem. 255, 7051; 1980).

Rossier and colleagues (Proc. natn. Acad. Sci. U.S.A. 77, 666; 1980) have shown that stress induces PRL synthesis and they believe that this arises from the stress-induced production of opiate peptides which are known to increase PRL levels.

More recent results (Denef et al. Nature 285,$243 ; 1980$ ) have suggested that the action of dopamine is not quite as simple as was previously thought. Although high levels $(\geqslant 1 \mu \mathrm{M})$ of dopamine do indeed reduce PRL, at much lower levels ( $\leqslant 1 \mathrm{nM}$ ) dopamine actually stimulates PRL production.

The nature of the interactions between prolactin and the steroid hormones is still not clear. Although oestradiol seems to stimulate PRL production, its metabolite, 2-hydroxyoestrone, produces a rapid decrease in PRL levels (Fishman and Tulchinsky Science 210, 73; 1980). The hydroxylating enzyme is inhibited by the opiate agonists which stimulate PRI release and is activated by antagonists which suppress PRL. This means that in vivo the opiates may affect PRL levels by their effect on the enzymes which metabolize the steroid hormones.

Clearly we still have some way to go in our attempts to understand how the levels of this interesting molecule really are regulated. 